\title{
Lactic Acidosis Associated with Metformin in Patients with Diabetic Kidney Disease: A Narrative Review
}

\author{
Fatkhu Rahman ${ }^{1}$, Syahrul Tuba ${ }^{1 *}$ \\ 1 Faculty of Military Pharmacy, The Republic of Indonesia Defense University, \\ Sentul, Indonesia
}

*Corresponding author: Syahrul Tuba,

Address $\quad$ : IPSC, Sentul, Bogor, West Java, 16810, Indonesia

E-Mail : syahrul.tuba@idu.ac.id

Orcid : :0000-0002-3082-8891

\begin{abstract}
Background: Diabetes Mellitus is a metabolite disorder with parameters of high blood sugar levels. In the management of diabetes can be used the drug metformin is the gold of choice to achieve a therapeutic effect and rarely causes side effects of the drug, but it still has debate view. However, if used in excessive doses for patients with kidney disease, it will be contraindicated with side effects such as lactic acidosis. Objective: This study aims to evaluate the side effect of Metformin for diabetic kidney diseases (DKD) patients. Method: This study used the Narrative Review Method that was obtained from 2011 to 2021, in the English language from PubMed, Google Scholar, and Cochrane Library. Results: Metformin is at the forefront of the treatment of type 2 diabetes mellitus (DM2). Metformin is likely to have lactic acidosis-related adverse effects in chronic kidney disease (CKD) patients, such as increased arterial lactate. Lactic acidosis is defined as an increase in arterial lactate with an indicator of more than five mmol/L and an arterial blood $\mathrm{pH}$ of less than 7.35. Metformin-induced lactate levels are considered to be below the parameters. DKD risk factors can be conceptually classified as several susceptibility factors, initiation factors, and developmental factors. The two most prominent risk factors are hyperglycemia and hypertension. Conclusion: Metformin can increase lactate levels in CKD patients but is still below the parameters of lactic acidosis. This study may have some weaknesses and requires further prospective research to validate the results.
\end{abstract}

Keyword: Diabetic, Chronic Kidney Disease, Metformin, Acidosis Lactate. 


\section{INTRODUCTION}

\section{Metformin is an antidiabetic drug that is thought to induce L.A}

Metformin is a drug that results in clear benefits concerning glucose metabolism and diabetes-related complications ${ }^{1}$, Being one of the most effective antidiabetic drugs to be an option in the treatment of type 2 diabetes ${ }^{2}$. Metformin is a biguanide drug to lower blood glucose levels by reducing glucose production in the liver, decreasing intestinal absorption, and increasing insulin sensitivity. Metformin is considered safe and well-tolerated. One of the side effects of Metformin is gastrointestinal, including nausea, vomiting, and diarrhea, with the percentage occurring in $30 \%$ of patients who use it. Very rarely occurs, such as experiencing chest discomfort, headache, diaphoresis, hypoglycemia, weakness, and rhinitis ${ }^{3}$.

The mechanism works Metformin by increasing peripheral glucose uptake, reducing intestinal glucose absorption, increasing insulin sensitivity, and decreasing fasting plasma insulin levels, thereby reducing blood glucose concentrations without causing hypoglycemia. In addition, Metformin can inhibit gluconeogenesis by activating AMP-activated protein kinase $(\mathrm{AMPK})^{4}$. Although Metformin has become the medicine of choice for the treatment of type 2 diabetes, due to the risk of lactic acidosis, some individuals may not be prescribed it ${ }^{5}$.

Lactic acid is produced in normal physiological processes and is a common finding in disease states ${ }^{6}$. Lactic acidosis is an acidosis characterized by excessive levels of lactic acid in the body. In this case, the buildup of acids, including lactic acid, can affect the balance of acids and bases $(\mathrm{pH})$ in the body. Lactic acidosis most likely contributes to worsening of the underlying comorbidities, and therefore severely contributes to mortality. Lactic acidosis is defined as an increase in arterial lactate with an indicator of more than five mmol/L and an arterial blood $\mathrm{pH}$ of less than $7.35^{7}$.

With a predominance of renal excretion, the risk of lactic acidosis in CKD patients is thought to be higher with metformin induced. Although the data noted a correlation between lactate levels and metformin dose, it is still insufficient to diagnose lactic acidosis. Even with patients receiving higher metformin doses, the results are too small to draw significant conclusions about whether higher metformin doses may be associated with lactic acidosis ${ }^{8}$.

\section{METHODS}

We have searched PubMed, Google Scholar, and Cochrane Library using the terms "lactic acidosis," "metformin," and "diabetic kidney disease" to retrieve all articles related to a possible link between Metformin and diabetic kidney disease, Method that obtained from 2011 to 2021, and in the English language. 


\section{DISCUSSION}

\section{Diabetes mellitus}

Diabetes mellitus is a group of metabolic diseases characterized by chronic hyperglycemia caused by insulin production, insulin action, or both ${ }^{9}$. The heart, blood vessels, eyes, kidneys, and nerves are the organs most affected by damage and failure due to chronic hyperglycemia $^{10}$. Based on the American Diabetes Association (ADA) in 2004, diabetes is divided into several sub-groups, namely type I diabetes (insulin-dependent) caused by immunemediated destruction of beta cells, leading to insulin deficiency. Idiopathic testing is a type of type 1 diabetes that has no known cause and is highly hereditary. Insulin secretion and insulin resistance are the causes of type II (non-insulin-dependent) diabetes. Gestational diabetes mellitus is a type of glucose intolerance that develops during or shortly after pregnancy. Generally, diabetes can be divided into two types, namely Diabetes Type I (IDDM) and Diabetes Type II (NIDDM) ${ }^{11}$.

\section{Metformin as therapy}

Metformin is thought to be the best drug for type 2 diabetes because it has the best initial pharmacological effect in decreasing blood glucose levels ${ }^{12}$. Metformin inhibits gluconeogenesis in T2DM patients, suppressing the increase in endogenous basal glucose synthesis. Long-term metformin treatment may result in a biochemical shortage of vitamin B12, which should be monitored regularly, especially in individuals with peripheral neuropathy or anemia ${ }^{13}$. Unfortunately, metformin use has been linked to lactic acidosis, a rare but potentially fatal adverse effect (LA). The most challenging aspect of metformin treatment in CKD patients is avoiding toxicity ${ }^{12}$. Metformin concentrations should be measured frequently to monitor therapy. In lactic acidosis, the frequency of lactic acidosis in metformin-treated patients is shallow, and it is numerically equivalent to the background rate in the type 2 diabetes population ${ }^{14}$.

The infusion of intravenous sodium bicarbonate is one type of handling for metformininduced lactic acidosis. Sodium bicarbonate has the benefit of restoring fluid balance, correcting acidosis and electrolytes but still has various drawbacks, including sodium-induced hypervolemia, exacerbation of intracellular acidosis, and hemoglobin oxygen depletion. Other therapies for metformin-associated lactic acidosis include hemodialysis, and Because Metformin has a high volume of distribution, hemodialysis removes only a tiny amount of the drug. Treatment with this strategy is very effective for addressing significant acidosis symptoms. When starting hemodialysis, a rebound effect is observed. Metformin accumulates 
in erythrocytes and intestines, where it produces and increases serum lactate levels. When serum lactate levels return to normal and lactic acidosis is resolved, it is advisable to continue hemodialysis. Metformin itself does not cause hypoglycemia, but it can cause hypoglycemia under certain conditions, such as fasting, sustained physical exercise, and renal failure. Intravenous glucose can be used to treat this problem. However, intracellular glucose metabolism leads directly to lactate production. Some authors have suggested that glucose injection may exacerbate lactic acidosis ${ }^{13}$.

The three types of risk factors for DKD include susceptibility factors (e.g., age, gender, race/ethnicity, and family history), precipitating factors (e.g., hyperglycemia and AKI), and developmental factors (e.g., hypertension, dietary variables, and obesity) are all aspects to consider. Hyperglycemia and hypertension are two of the most well-known risk factors ${ }^{15}$.

\section{Table 1.}

Risk factors for diabetic kidney disease

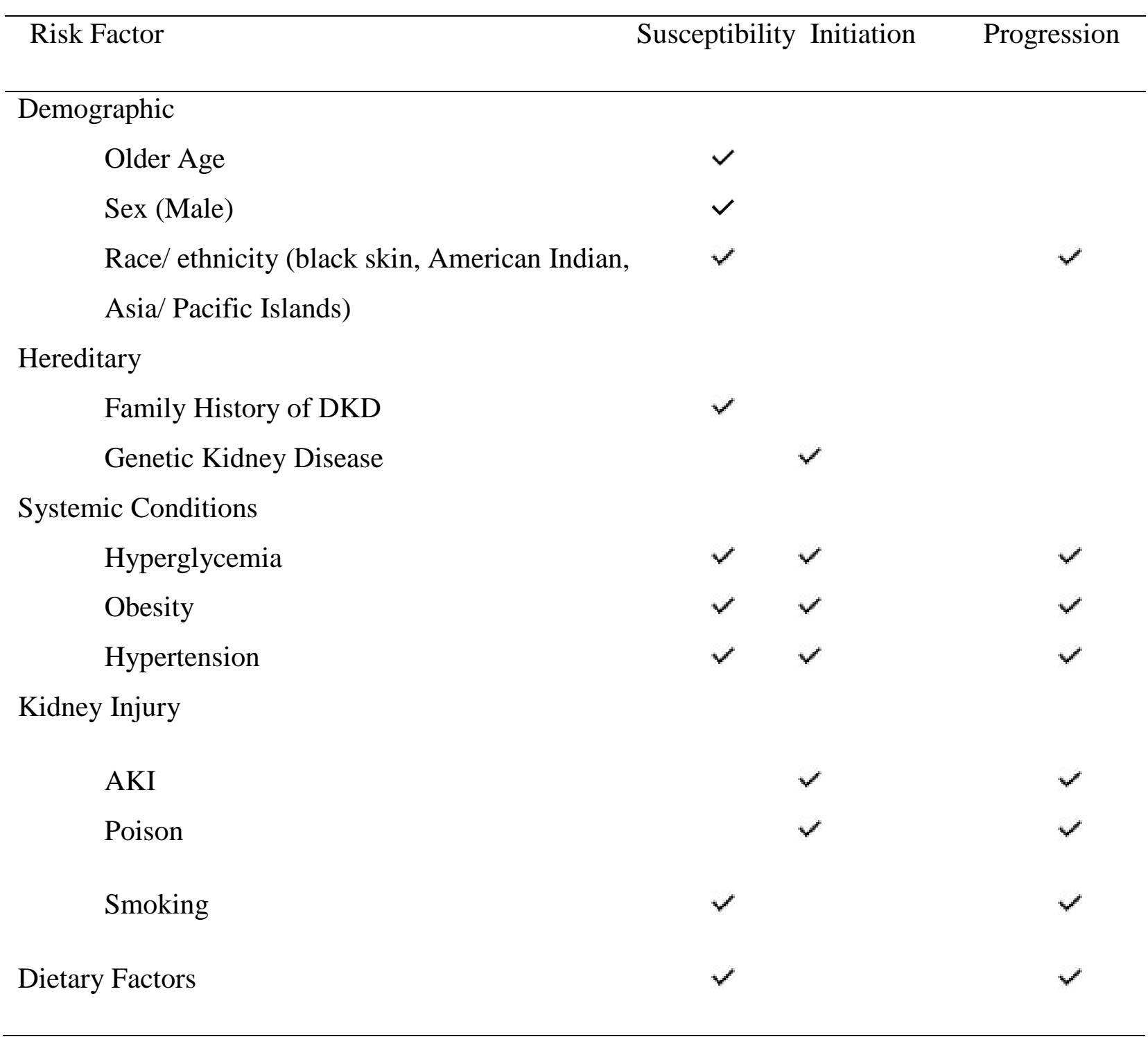




\section{High Protein Intake}

*DKD, diabetic kidney disease

\section{Condition of patients with CKD}

Chronic kidney disease (CKD) is a condition in which the kidneys are damaged or dysfunctional $^{16}$. A person is reported to have CKD if there are structural or functional abnormalities of the kidney for at least three months, Based on the KDIGO CKD recommendations (the British National Institute for Health and Care Excellence (NICE) CKD, 2014). Decreased eGFR (60 mL/min/1.73 m2), Albuminuria (urinary albumin-to-creatinine ratio [ACR] $30 \mathrm{mg} / \mathrm{g}$ [3 mg/mmol]) are some of the problems, Urinary sediment abnormalities such as hematuria, oval or fatty fat bodies, red or white blood cells, granular mold, and renal tubular epithelial cells. Any of these indicators must be present for at least three months ${ }^{17}$. Damage to kidney function as a result of a long-term disease is the cause of CKD. CKD can be caused by one of three disease processes: prerenal (lower renal perfusion pressure), intrinsic renal (vascular, glomerular, or tubular-interstitial pathology), or postrenal (increased renal perfusion pressure) (obstructive ${ }^{18}$. Diabetes and hypertension are the most common disorders that are the primary causes of CKD in different parts of the world ${ }^{19}$. Controlling blood pressure, optimal intake of protein and salt, modifying lifestyle and eating habits, and adhering to medication are all effective measures for slowing the progression of chronic kidney disease and lowering cardiovascular risk ${ }^{20}$.

\section{Mechanism of Lactic Acidosis}

Lactic acid (lactic acid/lactate) is a carbohydrate metabolism intermediate (intermediate product) generated mainly by white skeletal muscle, the interior of the kidney (renal medulla), the brain, skin, and erythrocytes ${ }^{21}$. This is a rare metformin adverse effect. However, it has significant consequences when used therapeutically or in excess. Lactic acidosis is divided into Type A (linked to or caused by a lack of oxygen in the tissues) and Type B (with adequate oxygen transport to tissues). Type B has three subtypes, namely B1 lactic acidosis caused by a disease (such as asthma and diabetes), B2 lactic acidosis induced by drugs or toxins (such as cyanide, Metformin, or epinephrine), and B3 lactic acidosis is caused by hereditary metabolic conditions. Lactate results from the degradation of pyruvate by the enzyme lactate dehydrogenase in anaerobic metabolism (this represents the product). It is produced at a rate of roughly one $\mathrm{mmol} / \mathrm{kg} / \mathrm{hr}$ in the intestine, skeletal muscle, brain, and circulating erythrocytes. The majority of lactate is taken up by the heart and recycled in three mechanisms. oxidation of 
pyruvate, conversion to glucose (Cori cycle), which can later be oxidized to CO2 (Krebs cycle), and transamination to alanine. Reduced oxygen supply to cells and tissues leads to impaired oxidative phosphorylation, increasing intracellular $\mathrm{NADH}$, where is a cofactor in pyruvate to lactate. Lactate is considered the end product of glycolysis due to hypoxia because of its close relationship with anaerobic metabolism ${ }^{22}$.

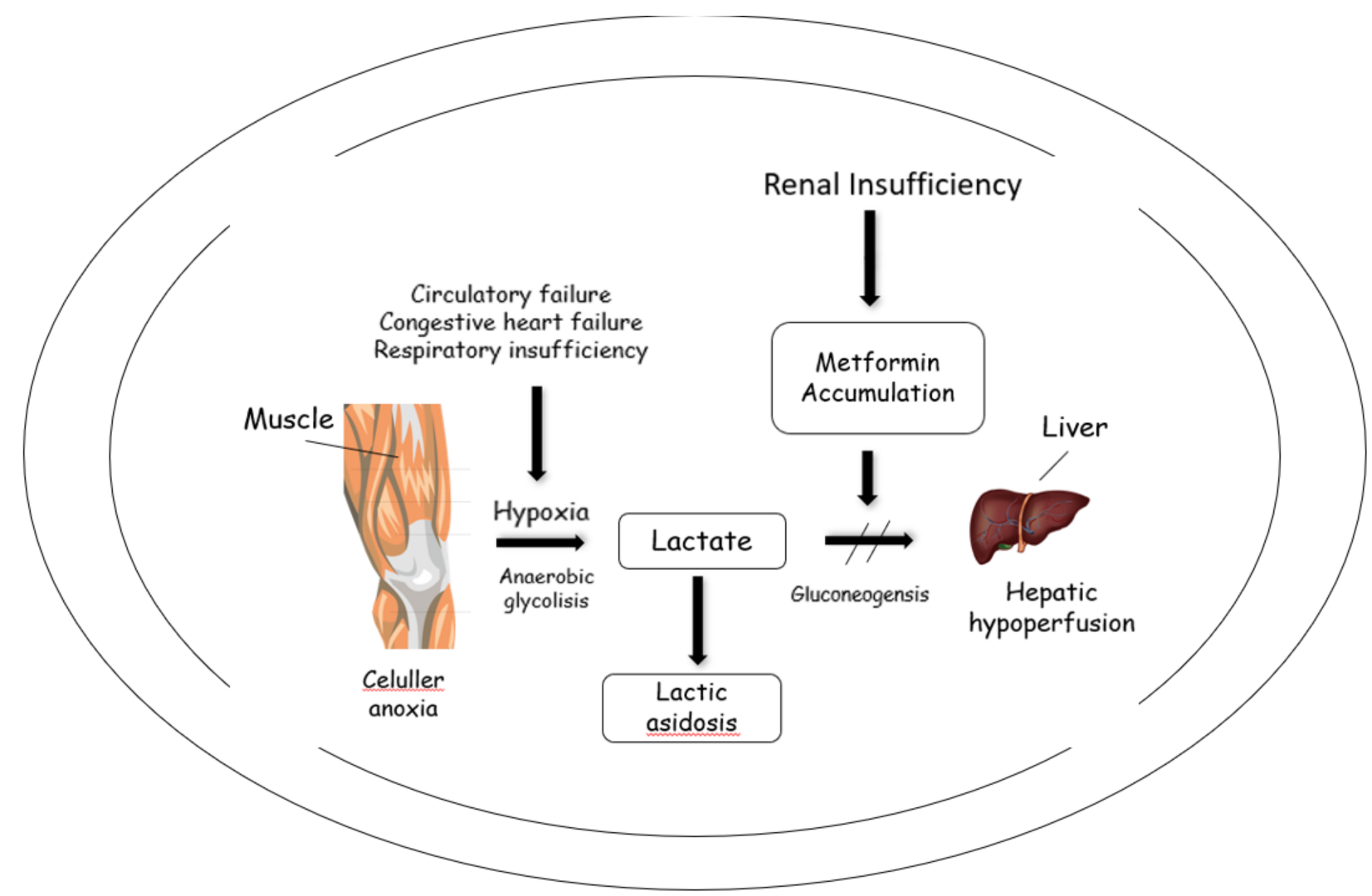

$<$ Figure 1. Mechanism of L. Acidosis $>$

Suspected to increase the risk of L.A associated with metformin therapy.

The most common risk factors for developing metformin-associated lactic acidosis include renal failure, chronic lung injury with hypoxia, pre-existing heart disease, and congestive heart failure, which are usually considered contraindications to treatment therapy. Increasing age can be associated with decreased renal function and increased comorbidities. Typical symptoms such as agitation, confusion, convulsions, dilated pupils, lethargy, absence of corneal reflexes, coma, and eventually death are common in patients with severe lactic acidosis. Dyspnea, lethargy, hypotension, hypothermia, confusion, and hemodynamic instability are causes of the development of lactic acidosis ${ }^{22}$. 


\section{CONCLUSION}

In several journals explained that Metformin could increase lactate levels in CKD patients. However, these parameters are still significantly lower than the benchmarks of lactic acidosis that have been established.

No consistent association between Metformin and lactic acidosis was found. However, observational studies may underestimate Metformin Associated Lactic Acidosis (MALA) risk that is indicated by poisoning. Although the renal dysfunction in this study was still healthier, patients who were prescribed Metformin explain the low-risk level for lactic acidosis.

\section{Acknowledgments}

The author would like to express special thanks to Nurhayati, Suwiarta Wangsa, Winda, Muhammad Abdu, Midwan Maulana Sahril, Faculty of Military Pharmacy Members, Prof. A. Octavian, Bantari Wisnu Kusuma Wardhani, Syahrul Tuba who have supported and assisted us in this project.

\section{Author's contributions}

FR as data analyst and main contributor in article writing, ST as supervisor, reviewer.

\section{REFERENCES}

1. Rena G, Hardie DG, Pearson ER. The mechanisms of action of metformin. Diabetologia 2017 60:9. 2017;60(9):1577-1585. doi:10.1007/S00125-017-4342-Z

2. Rajasurya V, Anjum H, Surani S. Metformin Use and Metformin-associated Lactic Acidosis in Intensive Care Unit Patients with Diabetes. Cureus. 2019;11(5). doi:10.7759/CUREUS.4739

3. Metformin - StatPearls - NCBI Bookshelf. Accessed May 21, 2021. https://www.ncbi.nlm.nih.gov/books/NBK518983/

4. Wang YW, He SJ, Feng X, et al. Metformin: A review of its potential indications. Drug Design, Development and Therapy. 2017;11:2421-2429. doi:10.2147/DDDT.S141675

5. Defronzo R, Fleming GA, Chen K, Bicsak TA. Metformin-associated lactic acidosis: Current perspectives on causes and risk. Metabolism. 2016;65(2):20-29. doi:10.1016/J.METABOL.2015.10.014 
6. Foucher CD, Tubben RE. Lactic Acidosis. StatPearls. Published online November 21, 2020. Accessed July 29, 2021. https://www.ncbi.nlm.nih.gov/books/NBK470202/

7. Foucher CD, Tubben RE. Lactic Acidosis. StatPearls Publishing; 2021. Accessed May 21, 2021. http://www.ncbi.nlm.nih.gov/pubmed/29262026

8. Bipi PK, George J, Gomathy S, Gracious N, Kumar S, Mohandas MK. Lactate levels and risk of lactic acidosis with metformin in diabetic kidney disease patients. Saudi journal of kidney diseases and transplantation: an official publication of the Saudi Center for Organ Transplantation, Saudi Arabia. 2017;28(6):1356-1361. doi:10.4103/1319-2442.220870

9. AT K, HM D. Diabetes mellitus: The epidemic of the century. World journal of diabetes. 2015;6(6):850. doi:10.4239/WJD.V6.I6.850

10. Chentli F, Azzoug S, Mahgoun S. Diabetes mellitus in elderly. Indian Journal of Endocrinology and Metabolism. 2015;19(6):744. doi:10.4103/2230-8210.167553

11. Asmat U, Abad K, Ismail K. Diabetes mellitus and oxidative stress-A concise review. Saudi Pharmaceutical Journal. 2016;24(5):547-553. doi:10.1016/J.JSPS.2015.03.013

12. Inzucchi SE, Lipska KJ, Mayo H, Bailey CJ, McGuire DK. Metformin in patientswith type 2 diabetes and kidney disease a systematic review. JAMA - Journal of the American Medical Association. 2014;312(24):2668-2675. doi:10.1001/jama.2014.15298

13. Salvatore T, Pafundi PC, Marfella R, et al. Metformin lactic acidosis: Should we still be afraid? Diabetes Research and Clinical Practice. 2019;157. doi:10.1016/j.diabres.2019.107879

14. Lipska KJ, Bailey CJ, Inzucchi SE. Use of metformin in the setting of mild-to-moderate renal insufficiency. Diabetes Care. 2011;34(6):1431-1437. doi:10.2337/dc10-2361

15. Alicic RZ, Rooney MT, Tuttle KR. Diabetic kidney disease: Challenges, progress, and possibilities. Clinical Journal of the American Society of Nephrology. 2017;12(12):2032-2045. doi:10.2215/CJN.11491116

16. Wouters OJ, O’Donoghue DJ, Ritchie J, Kanavos PG, Narva AS. Early chronic kidney disease: diagnosis, management and models of care. Nature reviews Nephrology. 2015;11(8):491. doi:10.1038/NRNEPH.2015.85 
17. Fraser SD, Blakeman T. Chronic kidney disease: identification and management in primary care. Pragmatic and Observational Research. 2016;7:21. doi:10.2147/POR.S97310

18. Vaidya SR, Aeddula NR. Chronic Renal Failure. The Scientific Basis of Urology, Second Edition. Published online July 16, 2021:257-264. Accessed July 27, 2021. https://www.ncbi.nlm.nih.gov/books/NBK535404/

19. Webster AC, Nagler E v., Morton RL, Masson P. Chronic Kidney Disease. The Lancet. 2017;389(10075):1238-1252. doi:10.1016/S0140-6736(16)32064-5

20. V J, G GG, K I, et al. chronic kidney disease: global dimension and perspectives. Lancet (London, England). 2013;382(9888):260-272. doi:10.1016/S0140-6736(13)60687-X

21. Indrayanti L, Mulyono H. Profil Asam Laktat Penderita Diabetes Mellitus Terkendali (Kontrol) Dan Tidak Terkendali (Kontrol). Indonesian Journal of Clinical Pathology and Medical Laboratory. 2018;14(3):97. doi:10.24293/ijcpml.v14i3.927

22. Strugaru AM, Botnariu G, Agoroaei L, Grigoriu IC, Butnaru E. METFORMIN INDUCED LACTIC ACIDOSIS-PARTICULARITIES AND COURSE. Vol 117. 